\title{
Cloud forecaster: Gizmo for Evaluation of Bulky Cloud Computing Surroundings to Propagate ICT based Education
}

\author{
Abhay Saxena ${ }^{\# 1}$, Chandrashekhar Patel ${ }^{* 2}$, Puneet Chandra Verma ${ }^{\# 3}$ Parth Gautam ${ }^{* 4}$ \\ ${ }^{\# 1,2,4}$ Department of Computer Science DSVV Haridwar, India \\ 1'abhaysaxena2009@gmail.com, ${ }^{2}$ shekharrockin1988@gmail.com \\ ${ }^{3}$ cvermapuneet@gmail.com, ${ }^{4}$ parth.gautam@dsvv.ac.in \\ ${ }^{\# 3}$ Department of Computer Science SIT Pithoragarh, India
}

\begin{abstract}
In recent year cloud computing become one of the rapid advancement field in computer science, which offers to access remote server for storing and retrieving our data. In this paper, we have used Cloudanalyst tool to determine load balancing factor, response time and cost of our data center for developing ICT based Education through Mobile Application. For analyzing our result, we have used 3 and 7 Data Centers and used Round Robin algorithm. For algorithm simulation, we have used five user bases named as UB1, UB2, UB3, UB4, UB5 and region R2. In our work, we basically concentrated on calculated Response Time and Data Center processing time for three and seven Data Centers. In our analysis, for three Data Centers we found that minimum Total Response Time is $42.23 \mathrm{~ms}$, maximum is 60.25ms and minimum data center processing time is $0.02 \mathrm{~ms}$, maximum is $1.35 \mathrm{~ms}$. For seven data

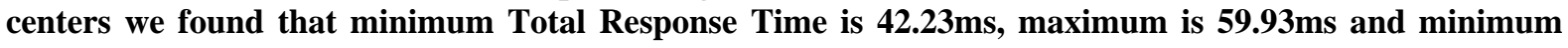
Data Center Processing time is $0.04 \mathrm{~ms}$, maximum is $1.40 \mathrm{~ms}$.
\end{abstract}

Keywords- Cloud analyst, Data center (DC), Virtual machines (VM), User bases (UB), Region, load balancing algorithms, service broker policy.

\section{INTRODUCTION}

Over the last few years, cloud computing services have become increasingly popular due to the evolving data centers and parallel computing paradigms [1]. A cloudis a type of parallel and distributed system consisting of a collection of interconnected and virtualized computers that are dynamically provisioned and presented as one or more unified computing resources based on service-level agreements established through negotiation between the service provider and consumers [2].

Virtualization is the principle aspect of cloud system. Using virtualization technique, cloud computing virtualized a single system into number of virtual systems. Basically a virtual machine is a software implementation of physical resource [3]. Fig. 1, shows that cloud computing is a combination of three important factors characteristics, deployment model and service model.

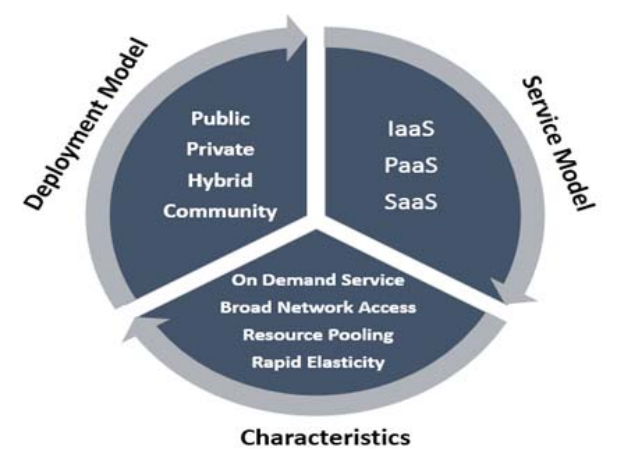

Fig. 1. Cloud Computing

\section{A. Cloud Analyst}

Cloud computing provides a tool for load balancing. Cloud analyst seems to be easy as it has a graphical user interface with which it seems like easy to use tool and also to have a level of visualization capability which is even better than just a tool-kit [4]. Cloud analyst separates simulation set up environment exercise and supports the modeller to focus on the parameters used for simulation purposes rather than the programming technicalities only [4]. It also supports a modeller to perform simulations continually by modifying the parameters easily, quickly and in very less time [4]. Cloud Analyst is an extension to cloudsim oriented simulator used for modeling and simulation of real cloud environment [5]. 


\section{B. Load Balancing}

Load balancing is relatively new technique that facilitates networks and resources by providing a maximum throughput with minimum response time [6]. As technology growing faster, there are huge amount of users on internet, for managing and fulfill their requirements, load balancer come in to picture which essentially ensure that they distribute workload equally to the all available server without any delay which help to accomplish a high user satisfaction, Maximum throughput with minimum response time [7]. We use Round Robin algorithms to distribute the load, check the response time and data center processing time.

\section{Round Robin Load Balancing Algorithm}

This is a load balancing algorithm which uses the Round robin concept. It selects the load randomly in case that some server is heavily loaded or some are lightly loaded. It utilizes the principle of time slices which divided time into multiple interval and each VM is given a particular time slice or time interval [8]. Round robin works on arbitrary selection of the VMs. It assigns requests to a list of existing VMs on a rotational basis. The first request is assigned to a VM selected arbitrarily from the group and then the Data Centre controller allots the requests in a circular order. When the VM is assigned the request, the VM is progressed to the end of the list [9]. In fig. 2 we have shown implementation of round robin algorithm on different Virtual Machines.

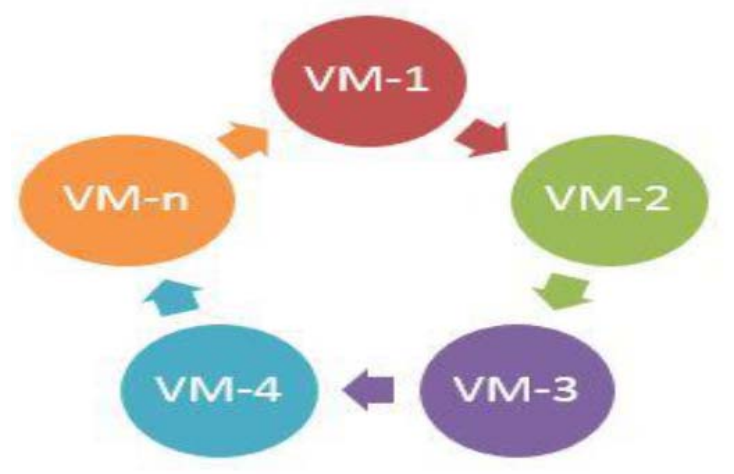

Fig. 2.Round Robin Load Balancing

\section{RESULTS \& ANALYSIS}

In this paper, we determine the Efficiency of Data Centers. For obtaining this efficiency, we use number of Data Centers 3 and 7 and use Round Robin algorithm. Fig. 3 shows the Region Boundaries in Cloud Analyst and TABLE-I describing the configuration details.

For algorithm simulation, we use five user bases named as UB1, UB2, UB3, UB4, UB5 and use region R2 for every 3 and 7 data centers. Cloud Analyst consist 3 main scenarios, first is Main Configuration, second is Data Center Configuration and third is advanced. Main configuration consists of User bases Configuration, Application Deployment Configuration and Service Broken Policy. Data Center Configuration consist the information about Data Centers and Physical Hardware Detail of Data Center. Advanced Tab consists the value of User grouping factor in User Bases, Request grouping factor in Data Centers, Executable instruction length per request and Load balancing policy across VM's in a single Data Center.

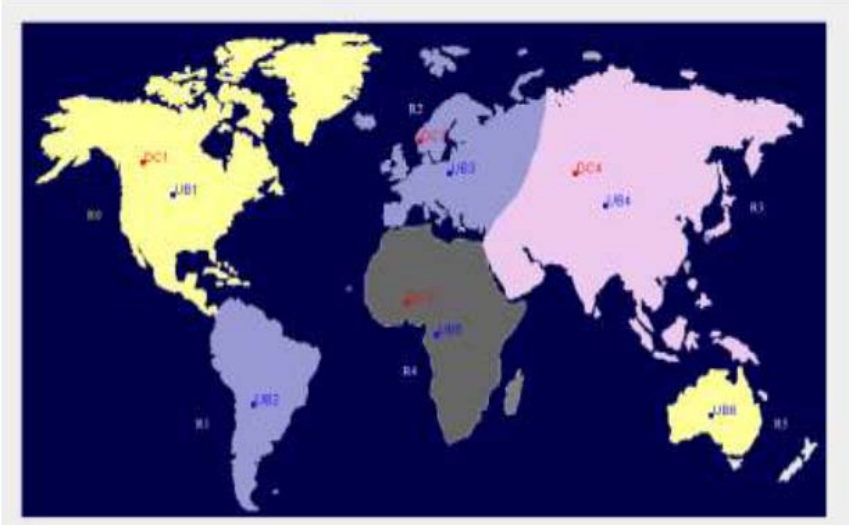

Fig. 3.Region Boundaries in Cloud Analyst 
TABLE I Configuration Details

\begin{tabular}{|l|l|}
\hline Parameter & $\begin{array}{l}\text { Value Used for THREE \& } \\
\text { SEVEN Data Centers }\end{array}$ \\
\hline UB Name & UB1, UB2, UB3, UB4, UB5 \\
\hline Region & 2 \\
\hline Request Per User Per Hour & 60 \\
\hline Data Size Per Request & 1000 \\
\hline Peak hour start(GMT) & 3 \\
\hline Peak hour end (GMT) & 9 \\
\hline Avg Peak Users & 1000 \\
\hline Avg Off Peak Users & 100 \\
\hline VM Image Size & $10000 \mathrm{MB}$ \\
\hline VM Memory & $512 \mathrm{MB}$ \\
\hline VM Bandwidth & $1000 \mathrm{bps}$ \\
\hline
\end{tabular}

\section{A. Results with Three Data Center}

Response Time by Region, Data Center Request Servicing Time, Overall Cost and Overall Response Time Summary are shown respectively in TABLE II, TABLE III, TABLE IV and TABLE V, when 3 Data Centers are used -

TABLE II Response Time by Region

\begin{tabular}{|l|l|l|l|}
\hline Userbase & Avg (ms) & Min (ms) & Max (ms) \\
\hline UB1 & 51.15 & 44.94 & 58.59 \\
\hline UB2 & 50.00 & 42.77 & 60.25 \\
\hline UB3 & 49.89 & 42.23 & 59.32 \\
\hline UB4 & 51.02 & 43.34 & 57.33 \\
\hline UB5 & 50.39 & 42.99 & 56.15 \\
\hline
\end{tabular}

In TABLE II, we have calculated response time by region and found that for UB 3 we have minimum Average and Minimum response time which is 49.89 and 42.23 respectively and for max it is UB4 having 57.33.

TABLE III Data Center Request Servicing Time

\begin{tabular}{|l|l|l|l|}
\hline Data Center & Avg (ms) & Min (ms) & Max (ms) \\
\hline DC1 & 0.69 & 0.02 & 1.29 \\
\hline DC2 & 0.80 & 0.03 & 1.35 \\
\hline DC3 & 0.76 & 0.02 & 1.30 \\
\hline
\end{tabular}

In TABLE III,we have calculated Average, Minimum and Maximum request service time of data center and found that $\mathrm{DC} 1$ has minimum request time for all three parameter which is $0.69 \mathrm{~ms}, .02 \mathrm{~ms}$ and $1.29 \mathrm{~ms}$ respectively.

TABLE IV Overall Cost

\begin{tabular}{|l|l|l|l|}
\hline Data Center & VM Cost \$ & Data Transfer Cost \$ & Total \$ \\
\hline DC2 & 0.80 & 0.81 & 1.61 \\
\hline DC1 & 0.80 & 0.76 & 1.56 \\
\hline DC3 & 0.50 & 0.84 & 1.34 \\
\hline
\end{tabular}

In TABLE IV,we have calculated overall cost of all datacenter and found that Datacenter 3 has minimum cost which is 1.34 . 
TABLE V Overall Response Time Summary

\begin{tabular}{|c|c|c|c|}
\hline Parameter & Average (ms) & Min. (ms) & Max. (ms) \\
\hline Total Response Time & 50.49 & 42.23 & 60.25 \\
\hline Data Center Processing Time & 0.75 & 0.02 & 1.35 \\
\hline
\end{tabular}

In TABLE V,we have shown summary the of Total Response Time and Data center processing time for Three Data Center. We already have shown this data through bar graph as shown in Fig. 4.

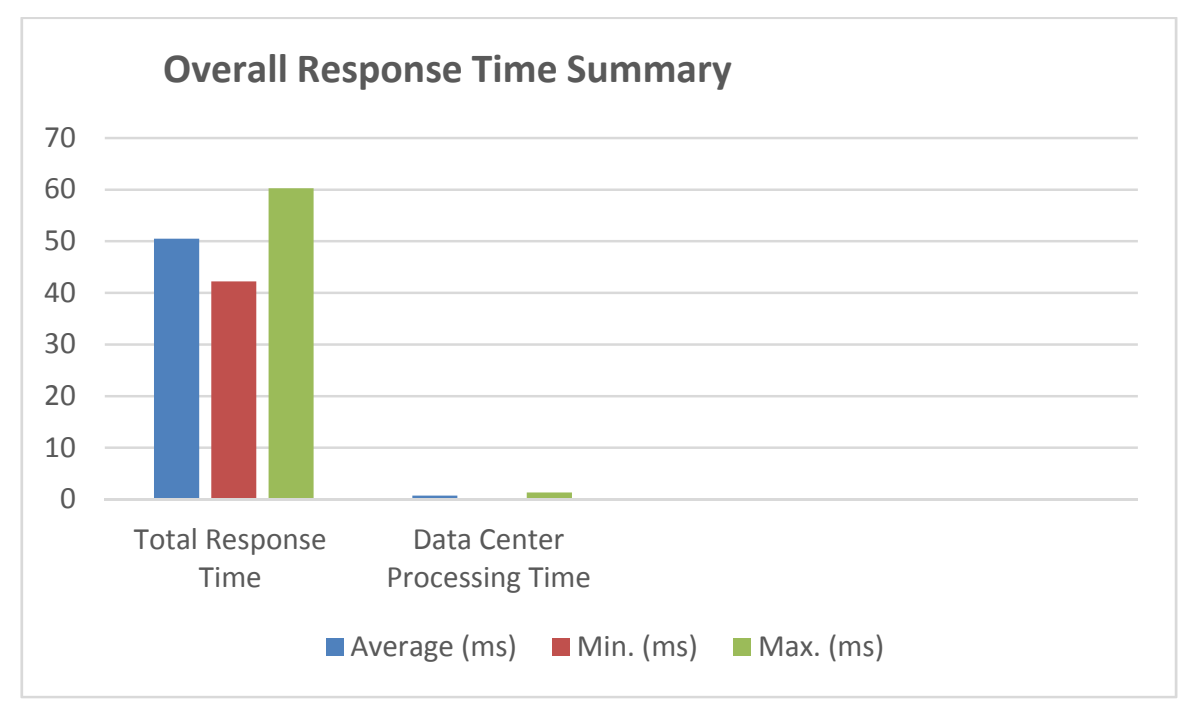

Fig. 4.Overall Response Time Summary for Three Data Center

\section{B.Results with Seven Data Center}

In below table we have found some results for seven Data Center.

TABLE VI Response Time by Region

\begin{tabular}{|c|c|c|c|}
\hline Userbase & Avg (ms) & Min (ms) & Max (ms) \\
\hline UB1 & 51.20 & 44.94 & 58.59 \\
\hline UB2 & 49.94 & 42.77 & 57.57 \\
\hline UB3 & 49.81 & 42.23 & 59.32 \\
\hline UB4 & 51.06 & 43.34 & 59.93 \\
\hline UB5 & 50.52 & 43.05 & 56.24 \\
\hline
\end{tabular}

In TABLE VI, we have calculated Average, Minimum, and maximum response time by the region. In our analysis we have found UB3 region has minimum Average response and UB4 has maximum response time.

TABLE VII Data Center Request Servicing Time

\begin{tabular}{|c|c|c|c|}
\hline Data Center & Avg (ms) & Min (ms) & Max (ms) \\
\hline DC1 & 0.74 & 0.06 & 1.30 \\
\hline DC2 & 0.81 & 0.06 & 1.29 \\
\hline DC3 & 0.66 & 0.05 & 1.29 \\
\hline DC4 & 0.76 & 0.06 & 1.34 \\
\hline DC5 & 0.80 & 0.11 & 1.33 \\
\hline DC6 & 0.69 & 0.04 & 1.40 \\
\hline DC7 & 0.80 & 0.07 & 1.29 \\
\hline
\end{tabular}

In TABLE VII,we have calculated Average, Minimum, and maximum request servicing time for each data center. In our analysis we have found Data Center3 has minimum Request Servicing Time and Data Center 6 has maximum Request Servicing Time. 
TABLE VIII Overall Costs

\begin{tabular}{|c|c|c|c|}
\hline Data Center & VM Cost \$ & Data Transfer Cost \$ & Total \$ \\
\hline DC2 & 0.80 & 0.50 & 1.30 \\
\hline DC1 & 0.80 & 0.46 & 1.26 \\
\hline DC4 & 0.80 & 0.36 & 1.16 \\
\hline DC3 & 0.80 & 0.34 & 1.14 \\
\hline DC6 & 0.80 & 0.34 & 1.14 \\
\hline DC5 & 0.80 & 0.50 & 1.30 \\
\hline DC7 & 0.50 & 0.46 & 0.96 \\
\hline
\end{tabular}

In TABLE VIII,we have calculated overall cost to implement each data center in physical manner.

TABLE IX Overall Response Time Summary

\begin{tabular}{|c|c|c|c|}
\hline Parameter & Average (ms) & Min. (ms) & Max. (ms) \\
\hline Total Response Time & 50.50 & 42.23 & 59.93 \\
\hline Data Center Processing Time & 0.76 & 0.04 & 1.40 \\
\hline
\end{tabular}

In TABLE IX, we have represented summary of Total response time and data center processing time for seven Data Center. We have also shown our results in bar graph for better analysis in fig.5.

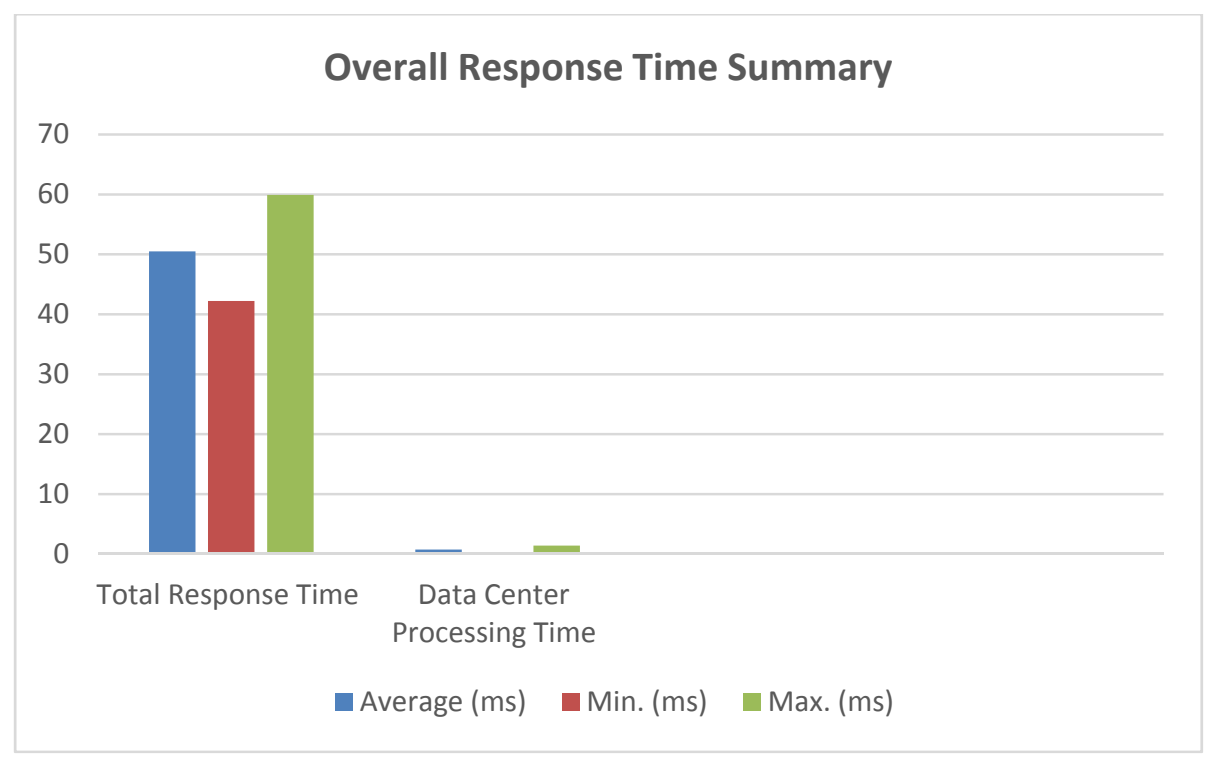

Fig. 5.Overall Response Time Summary for Seven Data Center

\section{CONCLUSION}

ICT based classes will improve the quality of education in the rural areas of India because it intends to meet flexibility and quickly changing software requirements for present and future ICT based applications. Another advantage of using cloud based model is that an operating system served by the cloud can be upgraded once then we need not to change on each individual platform.

In this paper, the performance of Round Robin is analyzed on small and large data centers with same regions. After concerning the minimum response time and data center processing time, we found that total minimum response time is same for both three and seven data centers, but there is a little difference between minimum data center processing time of both data centers. So, we can conclude that Round Robin algorithms is efficient for distributing the load, minimum response time and for minimum data center processing time over the large cloud based Data Centers.

\section{FUTURE SCOPE}

In future, we can analyze the efficiency of other load balancing algorithms like Weighted Round Robin with different set of data centers by using other load balancing algorithms and different service broker policies. 


\section{REFERENCES}

[1] Rimal BP, Choi E, Lumb I, A taxonomy and survey of cloud computing systems. In: The fifth international joint conference on INC, IMS and IDC, pp 44-51(2009).

[2] R. Buyya, C. S. Yeo, S. Venugopa.Market-oriented cloud computing: Vision, hype, and reality for delivering it services as computing utilities. In Proceedings of the $10^{\text {th }}$ IEEE International Conference on High Performance Computing and Communications (HPCC-08, IEEE CS Press,Los Alamitos, CA, USA) (2008).

[3] S.Angeles,20january 2014 [Online]. Available: http://www.businessnewsdaily.com/5791-virtualizationvs-cloud-computing.html. [Accessed 20 april 2016].

[4] Jeong H-Y, Park JH. An efficient cloud storage model for cloud computing environment. GPC 2012, (2012), 370-376.

[5] B. Wickremasinghe, "CloudAnalyst: A CloudSim-based Tool for Modelling and Analysis of Large Scale Cloud Computing Environments," 2009

[6] R. Shimonski. Windows 2000 \& Windows Server 2003 Clustering and Load Balancing.Emeryville.McGraw-Hill Professional Publishing, CA, USA (2003), p 2, 2003.

[7] Kaur, Rajwinder, and PawanLuthra. "Load Balancing in Cloud Computing."Proceedings of International Conference on Recent Trends in Information, Telecommunication and Computing, ITC. 2012.

[8] Nusrat Pasha, Dr. AmitAgrawal, Dr. Ravi Rastogi, "Round Robin Approach for VM Load Balancing Algorithm in Cloud Computing Environment", IJARCSSE, Volume 4, pages 34-39 Issue 5, May 2014.

[9] Domanal, S. G., \& Reddy, G. R. M. (2013, October). Load Balancing in Cloud Computing using Modified Throttled Algorithm. In Cloud Computing in Emerging Markets, 2013 IEEE International Conference on (pp. 1-5).IEEE. 